This article has been accepted for publication in a future issue of this conference proceedings, but has not been fully edited. Content may change prior to final publication. Citation information: DOI10.1109/ICECCME52200.2021.9590884, 2021 International Conference on Electrical, Computer, Communications and Mechatronics Engineering (ICECCME)

\title{
Review of Battery Management Systems
}

\author{
Mohamed Darwish \\ Brunel University \\ Department of Electronic and \\ Electrical Engineering \\ London, U.K \\ $\underline{\text { mohamed.darwish@brunel.ac.uk }}$
}

\author{
A. Janbey \\ London College UCK \\ Division of Engineering \\ London, U.K \\ a.janbey@1cuck.ac.uk
}

\author{
H. Amreiz \\ London College UCK \\ Division of Engineering \\ London, U.K \\ h.amreiz@1cuck.ac.uk
}

\author{
Christos C. Marouchos \\ Electrical Engineering, Computer Engineering and Informatics \\ Cyprus University of Technology \\ Limassol, Cyprus \\ christos.marouchos@cut.ac.cy
}

\author{
Stelios Ioannou \\ Electrical and Electronic Engineering, School of Sciences \\ University of Central Lancashire \\ Pyla, Larnaca, Cyprus \\ SIoannou2@uclan.ac.uk
}

\begin{abstract}
In this paper the authors look into different parts of Battery Management Systems (BMS) for rechargeable batteries. Compared to other chemistries, lithium batteries offer high energy density and cell voltage, which makes them the most attractive choice for electronic devices including EV and RES. However, lithium technology is vulnerable and highly susceptible to catastrophic failures which result in fire. Hence, the use of electronic safety designs is a must. BMS measure and monitor the battery state, allowing it to function in a safe way. BMS have a series of functions, but the state of charge (SoC) estimation is particularly observed. The estimation methodologies have advantages and disadvantages, and by better understanding them a BMS designer can choose which ones are better suited for the application that is developed for. The optimum BMS method will give the battery pack the needed protection, will keep the battery in a good functioning condition and will give an accurate prediction for the battery pack life.
\end{abstract}

Keywords - Battery Management Systems, State of Charge, Peukert's Equation..

\section{INTRODUCTION}

With mobile and portable devices having a bigger share of the market there is a need for an improved energy usage from the batteries that power them. Compared to other battery technologies, lithium batteries offer high energy density and cell voltage, which makes them the most attractive choice for electronic devices including EV and RES. However, lithium technology is vulnerable and highly susceptible to catastrophic failures which result in fire. Hence, the use of electronic safety designs, battery management systems (BMS), come into focus. BMS measure and monitor the battery state which include the state-of-charge (SOC), stateof-health (SOH), state-of-function (SOF), and state-oftemperature (SOT). Many publications as recorded by the elaborate review papers [1-5] have proposed different battery management implementations in a variety of configurations. Most have also charging algorithms implemented using ICs. With battery behaviour influencing the performance of BMS, it is one of the least understood parts of a charging system, so a better understanding can help with improvements. For battery management there is the need for a multitude of data, like discharge curves in different conditions, temperature, voltage and current and when the battery reaches an unsafe operating interval as defined by the manufacturer then it needs to be disconnected as a protection mechanism, as safety is one of the main concerns of manufacturers for liability reasons.
BMS designers need to understand all the processes involved and required, as well as the advantages and disadvantages of existing technologies, so that the proper algorithms and circuitry are selected. The purpose of the paper is to introduce the engineers to the topic under investigation. Therefore, this paper is organised as follows. Section II, offers an extensive battery technology overview, followed by battery mathematical modelling in section III. The reader is introduced to charging algorithms and state of charge in sections IV and V respectively. Battery charging rates (section VI) follow along with BMS operational conditions (section VII). Finally, cell balancing techniques are explained and analysed followed by discussion and future work.

\section{BATTERY TECHNOLOGY OVERVIEW}

Battery technologies are electrochemical devices that store energy. When a load is connected across the cathode and anode terminals then the chemical energy is converted to electrical. The characteristics of various battery technologies summarised on Table 1 were obtained or derived from [6-9]. As shown the battery technologies have been around since the 1950s. The most used technologies are Nickel-Cadmium (NiCad), Nickel Metal Hydride (NiMH), Sealed Lead Acid (SLA) and Lithium (Ion and Polymer). There is always a trade-off when choosing a battery technology, hence engineers should study and understand the various characteristics:

- Technology Age: Shows the maturity of the technology. The oldest technology (NiCad) shows that it has been excessively studied and it is well understood.

- Energy Density (Wh/kg): Indicates the amount of energy with respect to the battery's weight. For example, Lithium offers the highest energy density which means that it can store 3 times more energy than SLA and twice as much as the rest of the other technologies.

- Internal Resistance: Represents the technology's opposition (resistance) to the flow of electrons (electrical current). High resistance indicates high internal losses (low efficiency). Technologies such as alkaline with the highest internal resistance are limited to low power applications.

- Load Current: Battery technologies have limited load current capabilities which are determined by the 
This article has been accepted for publication in a future issue of this conference proceedings, but has not been fully edited. Content may change prior to final publication. Citation information: DOI10.1109/ICECCME52200.2021.9590884, 2021 International Conference on Electrical, Computer, Communications and Mechatronics Engineering (ICECCME)

chemistry and manufacturing processes. Engineers should take into consideration the load's required current and batteries highest current capability. The load current should be less than the battery's current to avoid power quality issues.

TABLE 1: Overview of Various Battery Technologies

\begin{tabular}{ccccc}
\hline \hline & NiCad & NiMH & SLA & Lithium \\
\hline \hline $\begin{array}{c}\text { Energy Density } \\
(\mathrm{Wh} / \mathrm{Kg})\end{array}$ & $40-60$ & $60-80$ & 30 & 165 \\
Internal Resistance & $100-300$ & $200-800$ & $<100$ & $300-500$ \\
$(\mathrm{~m} \Omega)$ & 1500 & 500 & $200-300$ & $500-1000$ \\
Cycle Life & 1.2 & 1.2 & 2 & 3.6 \\
Cell Voltage & $>2 \mathrm{C}$ & $0.5-1 \mathrm{C}$ & $0.2 \mathrm{C}$ & $2 \mathrm{C}$ \\
Load Current & -20 to +60 & -20 to +60 & -20 to +60 \\
Operating Temp & -40 to +60 & $\$ 70$ & $\$ 25$ & $\$ 100$ \\
$\left({ }^{\circ} \mathrm{C}\right)$ & $\$ 50$ & 1990 & 1970 & 1991 \\
Cost & 1950 & & &
\end{tabular}

Therefore, taking into consideration the characteristics and trade-off some examples of the market trends are as follows:

- NiCad: its high life cycle, low internal resistance, and high load current characteristics make it an attractive choice for power tools, two-way radios and biomedical instruments.

- $\quad$ SLA: despite the low energy density, their low price makes them attractive for applications where volume and weight is not an issue.

- Lithium: is the most expensive technology. However, with their high energy density and cell voltage, lithium technology is the most attractive choice for electronic devices where dimensions and weight are critical such as unmanned aerial vehicles (UAVs) also known as unmanned aerial systems (UAS). Market trends and new research show that Lithium batteries have dominated not only mobile applications but also the RE applications. Hence, lithium batteries will be primarily investigated in this study.

Scaled up secondary batteries are composed of multiple cells connected in series to achieve the desired voltage and connected in parallel to achieve the desired current required for high power applications. Such applications include satellites, automobiles, and RES. As always in engineering, Utopia does not exist. The battery pack matrix (series and parallel connections) suffers from increased internal temperature which results to reduced battery life and can also potentially lead to thermal runaway. In the case of lithium technology uneven heat transfer may result in fire. Heat variations lead to different internal resistances for different cells and even when identical cells are charged in parallel, the cell with the lowest impedance will receive higher charging current. Hence, the charging process is controlled by temperature and voltage safety circuitry. Furthermore, improved battery performance may result from controlling the temperature which is also known as Thermal management. Thermal management includes the process of cooling to prevent overheating of the battery pack whereas at low temperature losses are overcome by heating the pack. Commercially available cars such as the Toyota Prius use parallel-flow air-cooling whereas the Nissan Tino uses passenger air to cool down the batteries [6].



Fig. 1: Normalized Weight and Volume for Scaled-up Lithium Packs

Scaled-up battery performance suffer from the "packing factor" compared to the single cell. According to the application and the rigidity required (i.e electric vehicles, RES, military) then the energy density can be reduced by 20 $60 \%$ whereas volumetric energy density can be reduced by 80 and $140 \%$.

\section{BATTERy MATHEMATICAL MODELLING}

Mathematical models are evaluated based on accuracy, computational complexity, configuration and analytical insight.

- Physical - Detailed description of physical processes.

- Empirical - Equations with empirically fitted parameters.

- Abstract - Batteries represented as electrical circuits.

- Mixed models - Combination of the above.

Based on the application, design engineers have used these models for optimal power management algorithms as well as customizing power sources under volume and weight constraints.

\section{BATTERY CHARgING ALGORITHMS}

To have an effective control process a good charging algorithm is required. The monitoring of the energy conversion yields to higher charging efficiency. The charging process can have different current specifications. From the losses in the side-reactions there is a need to charge more than $100 \%$ in order to compensate and to have a resulting full charge. Low-rate charging has a rate of approximate $0.1 \mathrm{C}$ timed by a set timer. This method is time consuming and has a relatively simple charging algorithm. One of the simplest methods is by using a DC voltage with a resistor for limiting the current applied. Quick charging uses a $0.3 \mathrm{C}$ charge rate and because of this it takes significantly less to charge, with timers being also used for it. Fast charging uses at least $1 \mathrm{C}$ for the charge rate to have a short charge time, most times less than one hour, with the initial SoC being the most important factor in determining the charge time.

Lithium batteries need to be charged using Constant Current (CC) and Constant Voltage (CV) modes. The use of $\mathrm{CC}$ is mainly in the first part and then this is followed by $\mathrm{CV}$ and decreasing current once the maximum voltage is reached as shown on Fig.2. 
This article has been accepted for publication in a future issue of this conference proceedings, but has not been fully edited. Content may change prior to final publication. Citation information: DOI10.1109/ICECCME52200.2021.9590884, 2021 International Conference on Electrical, Computer, Communications and Mechatronics Engineering (ICECCME)

The impact of temperature on battery charging is considerable and it does influence the battery life cycle.

- $\quad$ Lithium: High temperature speeds up charging but affects battery lifespan. Also, it is very risky to charge below freezing temperatures.

- $\quad$ NiMH \& NiCd: High temperature causes lower charging rate.

- $\quad$ Lead-acid: High temperature causes lower voltage threshold.

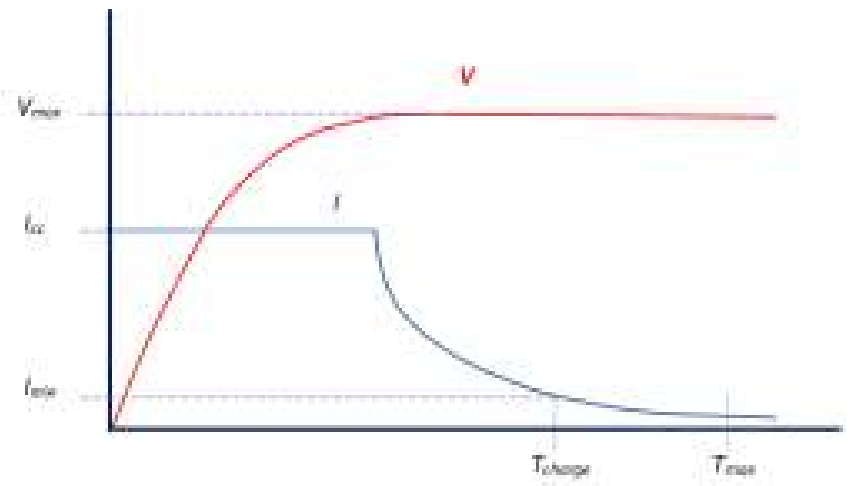

Fig. 3: A typical Lithium Charging I-V Characteristics

\section{State of Charge CAPAcity vs State of Charge ENERGY}

State of Charge (SoC) is known as the ratio of the capacity remaining to the total battery capacity. It should be noticed that SoC capacity $\left(\mathrm{SoC}_{\mathrm{C}}\right)$ is different from SoC energy $\left(\mathrm{SoC}_{\mathrm{E}}\right)$. As shown on Fig. 4, a $50 \% \mathrm{SoC}_{\mathrm{C}}$ is higher than $50 \% \mathrm{SOC}_{\mathrm{E}}$. In layman terms an EV will not travel the same distance as the first $50 \%$ because the shape of the curve. The area on the lefthand side is bigger than the area of the right-hand side at $50 \%$ $\mathrm{SoC}_{\mathrm{C}}$. Therefore, it is more relevant to look at the $\mathrm{SoC}_{\mathrm{E}}$ where the two areas are equal.

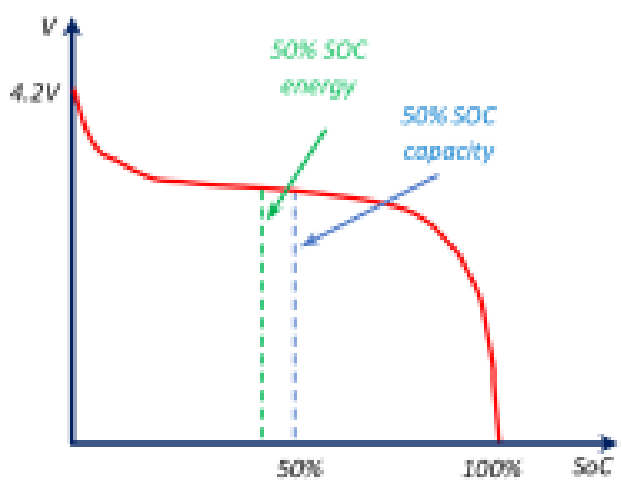

Fig. 4: SoC capacity vs SoC energy

The end or cut-off voltage for lithium technology according to the application and the manufacturer can be as low as $2.5 \mathrm{~V}$ whereas $3-3.3 \mathrm{~V}$ is the most widely used range. Voltages lower that the specified limit can permanently damage the battery as the chemical reaction becomes irreversible [6-9].

The main models for SOC are presented by equations 1 and 2 .

$$
C_{\text {Remain }}=C_{\text {Total }}-\int_{0}^{t} i(t) d t
$$

$$
C_{\text {Remain }}=e C_{\text {Total }}-\int_{0}^{t} i(t) d t
$$

Where $C_{\text {total }}$ is the battery initial capacity in Ah, $i$ is the load current in A and $e$ is the battery efficiency (loss of capacity).

The relationship between battery capacity and discharge current was initially noted by Schroder but it was not until 1897 when W. Peukert established a mathematical relationship for lead acid batteries which is known as Peukert's Equation or Peukert's Law [3], [6].

$$
C_{P}=I^{P} t
$$

where $I$ is the discharge current in Amperes (A), $t$ is runtime in hours (hr), $p$ is Peukert's exponent which is constant for a specific battery and depends on the battery chemistry, and $C_{p}$ is Peukert's battery capacity in Amperehours (Ah) which is assumed to be constant.

Researchers and experts agree that Peukert's equation account for battery losses / inefficiencies at higher discharge rates, and that the exponent value is constant and unique for every battery according to the battery type and usage. Furthermore, most research focuses on modelling and temperature compensation [3]. There is also some skepticism whether Peukert's equation calculates the total amount of energy obtained from a single discharge or from stages where the battery is at a specific discharge current, then left to rest, and then discharged a little bit more. An extensive literature review, as well as reading the actual paper [3] did not reveal any evidence on the topic. However, experimental data shown on Fig. 5, suggests that for single discharge, Peukert's equation is valid for discharge time in the range of 4 to 20 hours; at higher discharge rates it is not valid for lead-acid batteries. Whereas data from Fig. 6, show that the relationship is valid for lithium batteries.

Batteries today are completely different from 100 years ago; material and manufacturing advancements today enable lead acid batteries to achieve higher discharge rates ( 5 to 100C) and faster recovery time from deep discharges. These advancements also enable end of discharge voltages down to 1.37 volts per cell instead of 1.75 volts per cell [6], [9].

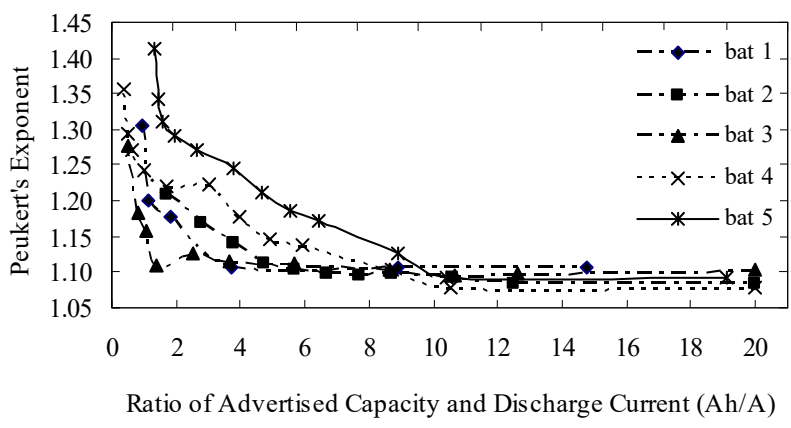

Fig. 5: Peurkert's Exponent as a function of the Ratio of Advertised Capacity and Discharge Current (Ah/A) for various Lead Acid Batteries.

Therefore, some new research proposes a reformulation of the relationship between battery capacity and discharge current with the introduction of a variable exponent which is a function of battery capacity and discharge current. The reformulated equation provides an accurate prediction of the runtime for single discharge applications using only the 
This article has been accepted for publication in a future issue of this conference proceedings, but has not been fully edited. Content may change prior to final publication. Citation information: DOI10.1109/ICECCME52200.2021.9590884, 2021 International Conference on Electrical, Computer,

Communications and Mechatronics Engineering (ICECCME)

battery name plate information such as capacity and the corresponding discharge time. The validation includes lead acid and lithium batteries.



Fig. 6: Peukert's Exponent Versus Ratio of Advertised Capacity and Discharge Current (Ah/A) for Lithium Batteries.

$$
t=\frac{t_{r e f}\left(C / t_{r e f}\right)^{a-b \ln (C / I)}}{I}{ }_{I}^{a-b \ln (C / I)}=t_{r e f}\left(\frac{C / t_{r e f}}{I}\right)^{a-b \ln (C / I)}
$$

where $a$ and $b$ are constant value which depend on the specific battery and as before $C$ is the nameplate capacity and $I$ is the discharge current. The values for $a$ and $b$ can be found experimentally using the "Half Hour Test" or obtained directly from the battery datasheet [6], [9].

Using the SOC models the reformulated approach was validated for lithium and lead-acid batteries including variable load current conditions which is more appropriate for most applications. The results showed an accuracy of $95 \%$.

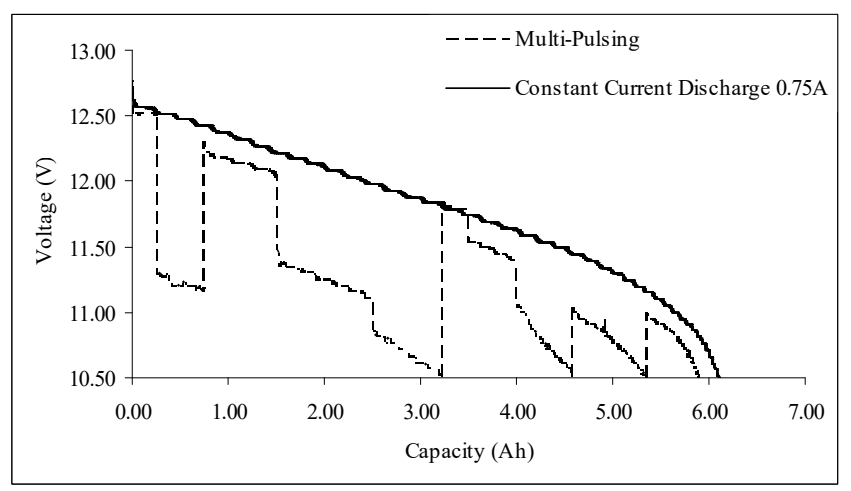

Fig. 7: Multi-Pulsing and Constant Current Discharge at 0.75A (1C).

The multi-pulsing discharge scenario applied to this battery was as follows: $0.75 \mathrm{~A}$ (or $1 \mathrm{C}$ ) for 0.33 hours, $7.6 \mathrm{~A}$ (10.1C) for 0.07 hours, $1.8 \mathrm{~A}(2.4 \mathrm{C})$ for 0.42 hours, $5.4 \mathrm{~A}$ (7.2C) for 0.19 hours, $7.2 \mathrm{~A}(9.6 \mathrm{C})$ for 0.1 hours, $0.75 \mathrm{~A}$ for 0.33 hours, 1.8 for 0.18 hours, 3.6 for 0.17 hours, $1.8 \mathrm{~A}$ for 0.42 hours and finally $0.75 \mathrm{~A}$ for 0.73 hours. At a current of $0.75 \mathrm{~A}$ gave a maximum estimated energy of $5.94 \mathrm{Ah}$ and a total delivered pulsed energy of 5.35Ah. Hence, this method suggests that after the described pulsing scenario then at a final discharge current of $0.75 \mathrm{~A}$ runtime it can continue for 0.887 hours whereas the experimental results show a runtime of 0.973 hours and a $9.73 \%$ difference.

\section{BATTERY CHARGING METHODS}

There are nine different charging methods which need to be well understood in order to fully appreciate the BMS. These can be classified as follows:

\section{A. Constant voltage}

A basic a DC power supply which may contains a transformer to step-down the voltage from the $\mathrm{AC}$ mains. It is mostly used in cheap battery chargers. Lead-acid and lithium-ion batteries usually use constant voltage chargers but in more complex form for the safety of users and also to protect the batteries.

\section{B. Constant current}

In constant current charging the voltage applied to the battery is varied in order to maintain s constant current flow. The charger is switched off once the battery voltage reaches the charge. Constant current is typically used in nickelcadmium and nickel-metal hybrid batteries.

\section{Pulsed charge}

It mainly supplies the battery with current pulses. The charging rate can be controlled by changing the duty cycle of the pulses. Each pulse usually takes about 1 second followed by 30 millisecond rest periods to allow the stabilisation of the battery chemical reaction.

\section{Taper charge}

In order to limit serious danger of damaging the cells if there is overcharging, in this mode the rate of charging is reduced as the battery voltage approaches the state of full charge.

\section{E. Negative pulse charge}

It is also called 'burp charging' or 'reflex charging'. This is used in conjunction with pulse charging. The main idea is to depolarise the cells for short time in order to allow any gas bubbles to dislodge. That can improve the charging mechanism.

\section{F. Trickle charge}

It is very effective to compensate for the self-discharge of battery cells. The charge rate depends on the frequency of the discharge. This mode is usually used in other modes once the battery is fully charged.

\section{G. Float charge}

This is used in emergency back-up systems where the battery and the load (usually critical loads) are connected in parallel.

\section{H. Random charging}

This is used when the energy used to charge the batteries is only available in some uncontrolled random way. This is usually the case in electric vehicle applications where regenerative brake control is always in use. Also, this is the case in solar panels where the charging can only be carried out where this is an irradiance.

\section{I-V-I charging}

In this mode of charging the battery is charged with constant rate current ' $I$ ' until the voltage reaches a predetermined value which just before the gassing occurs. This is called the bulk charge phase. After the that the charger switches into constant voltage phase ' $\mathrm{V}$ ' where the current will gradually drops until it reaches a predetermined value. In this phase the battery is charged at a slower rate. The final phase is the constant current mode 'I' where the voltage keeps rising to another predetermined value when the charger is switched off. 
This article has been accepted for publication in a future issue of this conference proceedings, but has not been fully edited. Content may change prior to final publication. Citation information: DOI10.1109/ICECCME52200.2021.9590884, 2021 International Conference on Electrical, Computer, Communications and Mechatronics Engineering (ICECCME)

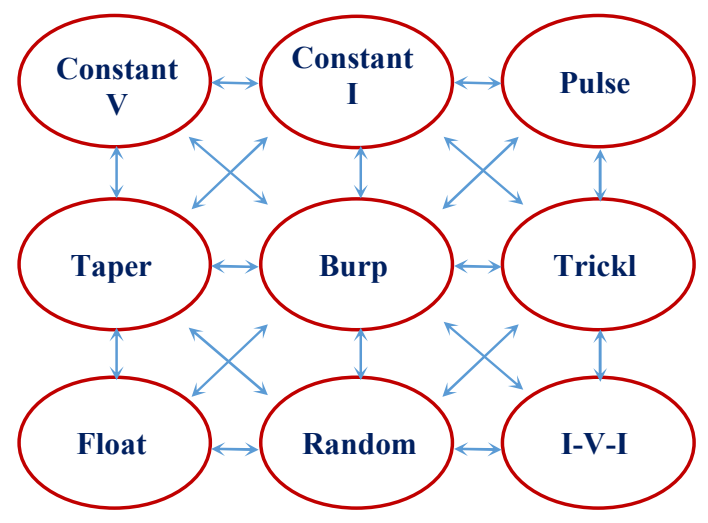

Fig. 8: Battery Charging methods

These nine methods are interrelated with each other's and in many cases, there is more than one method which is used at the same time as shown in Fig. 8.

\section{CHARGING RATES}

Typical battery charging rates can be classified into two categories: Slow charge and fast/quick charge. The different rates of charging is mainly depend on the requirement.

\section{A. Slow charging}

Slow charging is arguably the simplest and it is the most convenient way of charging EV. It should not also cause any battery overheating. Once the charging is complete the charging circuit should be disconnected. Different batteries will respond differently for overcharging. For example, $\mathrm{NiMH}$ cells can be damaged by trickle charging. Lithium ion cannot tolerate overcharging and the charging mechanism should be stopped immediately once the maximum voltage is reached. Lead acid can tolerate short duration of trickle charge. Nicads can be left on trickle charge for long periods of time. A typical slow charging time could be 10-16 hours charging at $0.1 \mathrm{C}$ rate.

\section{B. Fast / quick charging}

The issue with the high charging rate is the dangers of overheating (from $0.3 \mathrm{C}$ to 1.0 rate). This is very critical once the battery reaches full charge. Each cell has its own chemical and its own charging characteristic curve. The charger must be able to detect the end of the charging state. Some temperature cut-off is required to protect the battery from overheating during the charging process.

\section{VIII.BATTERY MANAGEMENT SYSTEM OPERATIONAL CONDITIONS}

For a good power management there is the need for added functions that deliver a balanced power distribution and minimal power usage. With energy management, specific functions have to be implemented for improved efficiency. The main purpose of a Battery Management System (BMS) is to make sure the battery is used in a way that provides maximum efficiency and minimum damage to the actual battery. Charging and discharging are the main processes that need to be closely monitored.

For applications like EVs and renewable energy storage there is a need for good designed battery storage systems. The battery management system (BMS) can be as important as the actual battery pack, in the sense that safe and efficient operation is desirable for the entire life of the system. There are a number of operational conditions that need to be considered for BMS development, as shown in Fig. 9.

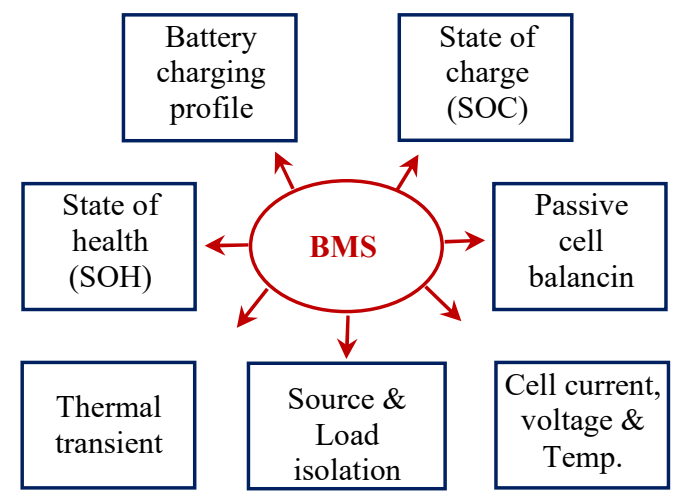

Fig. 9: Battery Management System diagram

\section{Cell Balancing}

Cell balancing, passive and active, is required mainly for pack stability [10]. When cells are charge/discharged unevenly then they exhibit different voltages which result in the event where the cell with the higher voltage charges the ones with lower voltages. Furthermore, as shown in Fig. 10 the weak cells will have an impact on the total run time of the battery pack which results in a fully depleted profile, despite the considerable amount of energy left unused in some of the cells.

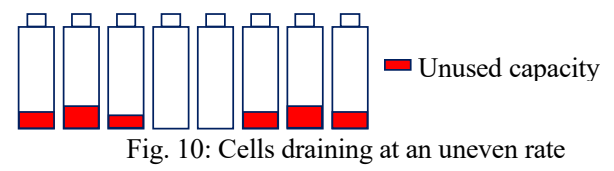

Passive cell balancing allows the battery stack as if every cell has the same capacity as the weakest cell. The health of each individual battery cell in the battery pack is based on its state of charge measurement (SoC). This measures the ratio of remaining charge in the cell to the cell capacity. The problem with passive cell balancing is that it allows all cells to appear to have the same capacity. The weakest battery cell will follow a charge and discharge cycle faster than a stronger cell. This could be a limiting factor in the system. To overcome this problem the high SoC cell is connected to a dissipative resistor (Fig. 11) so that charging can continue until all cells are equally fully charged. Passive balancing allows cells to have the same SoC and it provides low-cost mechanism for balancing the battery cells. However, it wastes energy because of the discharging resistor.

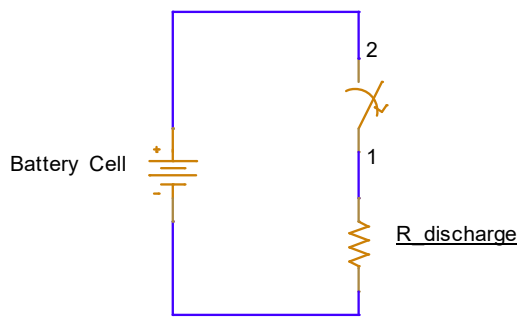

Fig. 11: Passive cell balancing

In active balancing, the unused charge is redistributed in a controlled manor from the stronger cells to the weaker cells. By using active balancing, the cells will all deplete at the same time all the way down to the minimum of $30 \%$. In this way 
This article has been accepted for publication in a future issue of this conference proceedings, but has not been fully edited. Content may change prior to final publication. Citation information: DOI10.1109/ICECCME52200.2021.9590884, 2021 International Conference on Electrical, Computer, Communications and Mechatronics Engineering (ICECCME)

the capacity of each cell is more efficiently utilised. This applies to both charging and discharging process.

A cell-balancing circuit for a series-connected lithium battery string is illustrated in Fig.12. The circuit consists of two networks: The bridge network with resonant tank and the cell access network with battery string.

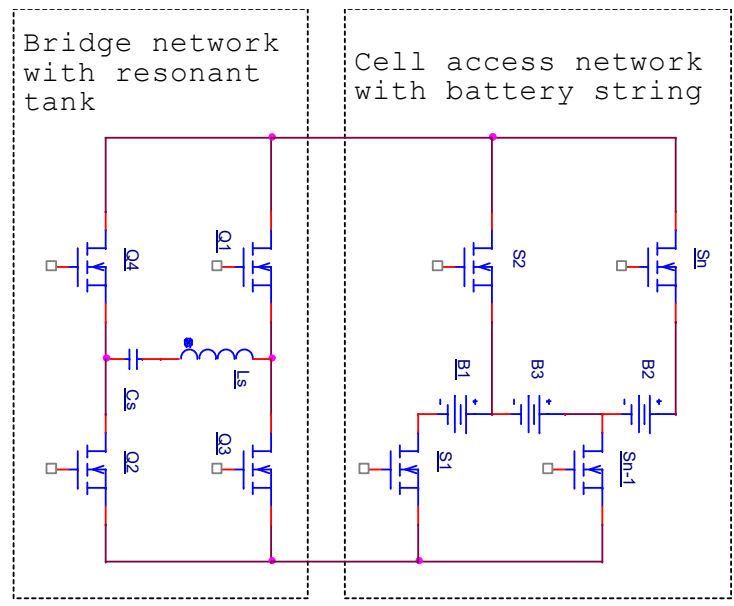

Fig. 12: Active cell balancing

\section{TEMPERATURE MONITORING}

The next important parameter is the temperature monitoring. Lithium batteries can provide large amount of current while maintaining constant voltage which can lead to a thermal dynamic condition and that can cause the battery to catch fire.

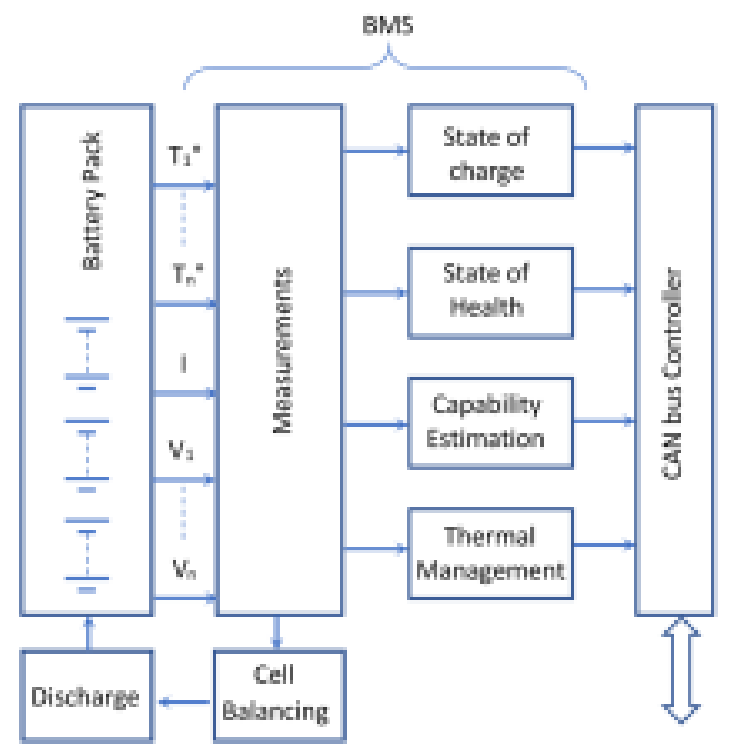

Fig. 13: Connection between CAN bus and BMS

The construction of the battery is highly volatile, and the temperature monitoring is not just for safety but it can also suggest if the temperature condition is suitable for battery charging or not. To measure the temperature of the battery, temperature sensors are used, generally thermistors are used as temperature sensors and they are generally temperature dependent resistors. Whenever there is a change in temperature, the resistance of the thermistors change and the BMS calculates the temperature rise accordingly. The BMS acts as a log-book to calculate the State of Health $(\mathrm{SoH})$ and other parameters of the battery, so for that purpose the BMShas to take the data accordingly to time so it should be working even if the EV is not ON. However, this may consume excess power from the battery itself and for this reason, a real time clock should be integrated within the BMS which consumes very little power. Fig. 13 illustrates the link between the measurement unit and the state of charge, state of health, capability estimation and thermal management on one hand and the CAN bus controller on the other hand.

\section{XI. . DISUSSION AND FUTURE WORK}

Battey management systems play an important role in monitoring the battery state. According to the battery technology the BMS regulates and maintains the proper battery operation within the manufacturer specifications, over and cut-off voltages, current, temperature, charge and discharge rates, etc.

Cell balancing, passive and active, is required mainly for pack stability. In active cell balancing system the charge is redistributed in both the charging and discharging phases. In passive cell balancing system the extra charge is dissipated in external resistor. Although passive balancing is more cost effective, but the active balancing is more efficient although it requires more complex circuit. SoC capacity differ from the SoC energy and it is important for the BMS to monitor the SoC energy and not the SoC capacity.

For future work, the authors will include hybrid systems composed of battery and a super-capacitor. Furthermore, additional energy and power systems should be included such as fuel-cells. Finally, it is suggested that the efficiency is examined as well as modulation processes and harmonics.

\section{REFERENCES}

[1] Park, S., Ahn, J., Kang, T. et al. Review of state-of-the-art battery state estimation technologies for battery management systems of stationary energy storage systems. J. Power Electron. 20, 1526-1540 (2020)

[2] M. A. Hannan, M. M. Hoque, A. Hussain, Y. Yusof and P. J. Ker, "State-of-the-Art and Energy Management System of Lithium-Ion Batteries in Electric Vehicle Applications: Issues and Recommendations," in IEEE Access, vol. 6, pp. 19362-19378, 2018

[3] Shreyas Maitreya;Himani Jain;Priyanka Paliwal, "Scalable and Decentralized Battery Management System for Parallel Operation of Multiple Battery Packs", IEEE conference in Innovations in Energy Management and Renewable Resources, 2021

[4] Xiangjun Li; Rui Ma; Lijun Wang; Shangxing Wang; Dong Hui, "Energy Management Strategy for Hybrid Energy Storage Systems with Echelon-use Power Battery", IEEE International Conference on Applied Superconductivity and Electromagnetic Devices (ASEMD), 2021.

[5] Jiguang Xue;Chunsheng Yan; Dan Wang;J un Wang; Jun Wu; Zehua Liao, "Adaptive Dynamic Programming Method for Optimal Battery Management of Battery Electric Vehicle", IEEE 9th Data Driven Control and Learning Systems Conference (DDCLS), 2020

[6] S. Ioannou, "Discrete Linear Constrained Multivariable Optimization for Power Sources of Mobile Systems", PhD Dissertation, Electrical Engineering, University of South Florida, November 2008.

[7] $\mathrm{S}$ Ioannou, $\mathrm{K}$ Dalamagkidis, KP Valavanis, EK Stefanakos, "Improving Endurance and Range of a UGV with Gimballed Landing Platform for Launching Small Unmanned Helicopters", Journal of Intelligent and Robotic Systems 53 (4), 399-416, 2008

[8] S. Ioannou, K. Dalamagkidis, K. P. Valavanis, E. K. Stefanakos and P. H. Wiley, "On Improving Endurance on Unmanned Ground Vehicles: The ATRV-Jr Case Study", 14th IEEE Mediterranean Conference on Control and Automation, June 28-30, 2006.

[9] S. Ioannou, K. Dalamagkidis, K. P. Valavanis, E. K. Stefanakos and P. H. Wiley, "Battery Capacity and Discharge Current Relationship for Lead Acid and Lithium Batteries", 24th IEEE Mediterranean Conference on Control and Automation (MED16), Greece, 2016.

[10] Kevin Scott and Sam Nork, "Active Battery Cell Balancing"; Analogue Devices, 2019. 